\title{
Angular momentum transport by internal gravity waves
}

\section{Wave excitation by core convection and the Coriolis effect}

\author{
F. P. Pantillon ${ }^{1,2}$, S. Talon ${ }^{2}$, and C. Charbonnel ${ }^{1,3}$ \\ ${ }^{1}$ Geneva Observatory, University of Geneva, ch. des Maillettes 51, 1290 Sauverny, Switzerland \\ e-mail: pantillon@astro. umontreal.ca \\ 2 Département de Physique, Université de Montréal, Montréal PQ H3C 3J7, Canada \\ e-mail: talon@astro.umontreal.ca \\ ${ }^{3}$ Laboratoire d'Astrophysique de Toulouse et Tarbes, CNRS UMR 5572, OMP, Université Paul Sabatier 3, 14 Av. E. Belin, \\ 31400 Toulouse, France \\ e-mail: Corinne.Charbonnel@obs.unige.ch
}

Received 13 June 2007 / Accepted 18 July 2007

ABSTRACT

\begin{abstract}
Context. This is the third in a series of papers that deal with angular momentum transport by internal gravity waves. We concentrate on the waves excited by core convection in a $3 M_{\odot}$, Pop I main sequence star.

Aims. Here, we want to examine the role of the Coriolis acceleration in the equations of motion that describe the behavior of waves and to evaluate its impact on angular momentum transport.

Methods. We use the so-called traditional approximation of geophysics, which allows variable separation in radial and horizontal components. In the presence of rotation, the horizontal structure is described by Hough functions instead of spherical harmonics.

Results. The Coriolis acceleration has two main effects on waves. It transforms pure gravity waves into gravito-inertial waves that have a larger amplitude closer to the equator, and it introduces new waves whose restoring force is mainly the conservation of vorticity. Conclusions. Taking the Coriolis acceleration into account changes the subtle balance between prograde and retrograde waves in nonrotating stars. It also introduces new types of waves that are either purely prograde or retrograde. We show in this paper where the local deposition of angular momentum by such waves is important.
\end{abstract}

Key words. hydrodynamics - turbulence - waves - methods: numerical - stars: interiors - stars: rotation

\section{Introduction}

Internal gravity waves (IGWs) have received growing attention in the past 15 years as a source of angular momentum redistribution, after the suggestion by various authors that they could be responsible for the Sun's quasi-flat rotation profile (Schatzman 1993; Zahn et al. 1997; Kumar \& Quataert 1997). Charbonnel \& Talon (2005) convincingly show that IGWs can play a major role in carrying angular momentum from the rapidly rotating core, left after the Sun's original contraction, to the surface convection zone that is continuously spun down by magnetic braking. In self-consistent evolutionary models that also takes into account the transport of angular momentum and chemical species by meridional circulation and shear turbulence, they also showed that the associated destruction of lithium is compatible with the destruction measured in the Sun. In the case of F dwarfs, Talon \& Charbonnel (2003) show that the combination of surface breaking for stars with an effective temperature $T_{\text {eff }} \lesssim 6900 \mathrm{~K}$ and the appearance of IGWs at a temperature $T_{\text {eff }} \lesssim 6600 \mathrm{~K}$ when the surface convection zone is thick enough can together explain the existence of the so-called lithium dip discovered by Wallerstein et al. (1965).

Now the question arises as to whether IGWs generated by a convection core also play a role in the evolution of massive stars ${ }^{1}$. Indeed, rotational mixing is now admittedly a major

${ }^{1}$ By massive stars we mean objects for which central hydrogen burning occurs in a convection core, i.e., stars with $M_{\text {ini }} \gtrsim 2.2 M_{\odot}$ at solar metallicity. ingredient in such objects (see e.g. Maeder \& Meynet 2000; Heger et al. 2000), and the existence of another major source of angular momentum redistribution within the star must be examined.

However, as a general rule, massive stars are fast rotators. Here, fast must be understood in comparison with the wave frequencies that dominate angular momentum redistribution in stars; that is, $\sigma \approx 1 \mu \mathrm{Hz}$ (Talon \& Charbonnel 2005). Our goal in this paper is to establish how wave properties are altered by rotation in preparation for full calculations of angular momentum redistribution in massive stars. Section 2 is devoted to examining the (horizontal) eigenfunctions in the presence of rotation, while Sect. 3 presents the results on angular momentum transport itself.

\section{Eigenfunctions in rotating stars}

\subsection{The traditional approximation}

In the study of internal waves in the presence of rotation, two effects should be considered: the star's distortion caused by the centrifugal force, and the modification to the momentum equation by the Coriolis acceleration. In 1D modeling, the stellar distortion can be accounted for by calculating an average centrifugal force that locally reduces gravity. This effect is quite important at the stellar surface where the ratio $r^{3} \Omega^{2} / G M_{\mathrm{r}}$ is at its maximum, and it modifies the location of the star in the HR diagram (see e.g. the ZAMS position of stars of various velocities in Fig. 5 of Talon et al. 1997). It does however have a minor impact on stellar evolution, since it modifies the physical 
conditions near the stellar core only slightly (see e.g. Maeder $\&$ Meynet 2000). In this work, we will neglect this term. The Coriolis acceleration, on the other hand, has a direct effect on the oscillations and modifies the momentum equations for the displacement $\boldsymbol{\xi}$, which become

$$
\begin{aligned}
& -\sigma^{2} \xi_{\mathrm{r}}-\underbrace{2 \mathrm{i} \sigma \Omega \sin \theta \xi_{\phi}}=-\frac{1}{\rho} \frac{\partial}{\partial r} P^{\prime}-\frac{\rho^{\prime}}{\rho} g \\
& -\sigma^{2} \xi_{\theta}-2 \mathrm{i} \sigma \Omega \cos \theta \xi_{\phi}=-\frac{1}{\rho r} \frac{\partial}{\partial \theta} P^{\prime} \\
& -\sigma^{2} \xi_{\phi}+\underbrace{\mathrm{i} \sigma\left(r \frac{\mathrm{d} \Omega}{\mathrm{d} r}+2 \Omega\right) \sin \theta \xi_{\mathrm{r}}}_{-\frac{1}{\rho r \sin \theta} \frac{\partial}{\partial \phi} P^{\prime}}+2 \mathrm{i} \sigma \Omega \cos \theta \xi_{\theta}=
\end{aligned}
$$

where $\sigma$ is the wave frequency, and other symbols have their usual meaning. Here, we have neglected variations in the gravitational potential $\left(\Phi^{\prime}=0\right.$, Cowling 1941), which is particularly justified for gravity waves that have mostly horizontal displacements. This equation was given in Talon (1997), and it differs from the one used by other authors (see below) only by the derivative in $\Omega$.

To proceed further, we make a simplification in our system of Eqs. (1)-(3) and neglect the horizontal component of the rotation vector $\Omega_{\mathrm{h}}=\Omega \sin \theta$. In the case of Eq. (1), this is justified by comparing this term with the vertical gradient of the pressure perturbation. In Eq. (3), we invoke the dispersion relation of gravito-inertial waves (see e.g. Unno et al. 1989)

$\sigma^{2}=\frac{N^{2} k_{\mathrm{h}}^{2}+(2 \boldsymbol{\Omega} \cdot \boldsymbol{k})^{2}}{k^{2}}$,

which in the case $\sigma^{2},(2 \Omega)^{2} \ll N^{2}$ (the condition for the approximation to be valid), yields

$k_{\mathrm{r}}^{2} \gg k_{\mathrm{h}}^{2}$.

This is similar to the result obtained for pure gravity waves. Since these low-frequency oscillations are quasi-incompressible, Eq. (5) is equivalent to

$\xi_{\mathrm{h}}^{2} \gg \xi_{\mathrm{r}}^{2}$,

whence the neglect of the second term $\left(\propto \xi_{\mathrm{r}}\right)$ of Eq. (3). This is known as the traditional approximation, which is well known in geophysics (see e.g. Eckart 1960), and it was used for the first time in an astrophysical context by Berthomieu et al. (1978).

In this case, wave eigenfunctions can be separated into radial and horizontal components. For the radial displacement, for example, one gets ${ }^{2}$

$\xi_{\mathrm{r}}(r, \theta, \phi, t)=\xi_{\mathrm{r}}(r) \Theta(\theta) \mathrm{e}^{\mathrm{i} i \phi} \mathrm{e}^{\mathrm{i} \sigma t}$.

The longitudinal eigenfunction is no longer given by an associate Legendre polynomial, as in the non rotating limit, but obeys instead a new differential eigenvalue problem of the form

$\mathcal{L}_{v}[\Theta(\theta)]=-\Lambda \Theta(\theta)$,

\footnotetext{
${ }^{2}$ Note that here we are using the convention of negative $m$ for prograde modes, contrary to what has been used in previous papers.
}

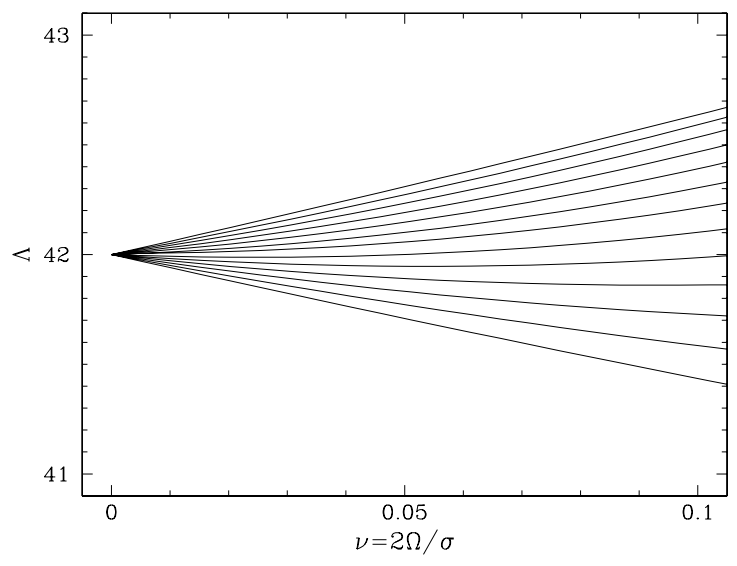

Fig. 1. Eigenvalues of Laplace's tidal equation when rotation is slowly increased. Illustrated modes correspond to the $\ell=6$ non-rotating case.

where

$$
\begin{aligned}
\mathcal{L}_{v}=\frac{\mathrm{d}}{\mathrm{d} x} & \left(\frac{1-x^{2}}{1-v^{2} x^{2}} \frac{\mathrm{d}}{\mathrm{d} x}\right) \\
& -\frac{1}{1-v^{2} x^{2}}\left(\frac{m^{2}}{1-x^{2}}+m v \frac{1+v^{2} x^{2}}{1-v^{2} x^{2}}\right) .
\end{aligned}
$$

In this equation, we use the rotation parameter

$v \equiv \frac{2 \Omega}{\sigma}$

and define $x=\cos \theta$. The azimuthal order takes integer values $m=0, \pm 1, \pm 2, \ldots$ This equation, known as Laplace's tidal equation, was originally derived in the context of terrestrial tides. It forms a Sturm-Liouville problem, ensuring the existence of a base of orthogonal eigenfunctions. These were described in the geophysical context by Hough (1898), and the first numerical solution was calculated by LonguetHiggins (1968). More recently, they have been examined in the context of neutron stars (Bildsten et al. 1996) and in studies of low-frequency oscillations in rotating stars (Lee \& Saio 1997; Daszyńska-Daszkiewicz et al. 2007, and references therein). We wrote a relaxation code to solve this equation numerically (see Press et al. 1992). A similar procedure is also described in Lee \& Saio (1997).

The Hough function $\Theta_{k m}(v, \cos \theta)$ is associated with the eigenvalue $\Lambda_{k m}(v)$. Following Lee \& Saio (1997), the order $k=0, \pm 1, \pm 2, \ldots$, which does not explicitly appear in Eq. (9), is such that $\Lambda_{k+1, m}(v)>\Lambda_{k m}(v)$. In the $v=0$ limit, Eq. (8) is identical to Legendre's equation with $k=\ell-|m|$ and $\Lambda_{k m}=\ell(\ell+1)$. Slowly increasing $v$, we first lift the degeneracy in $\Lambda$ (see Fig. 1). Increasing rotation confines eigenmodes toward the stellar equator (see Fig. 2).

In the case of more rapid rotation (i.e., $\sigma \lesssim 2 \Omega$ ), mode organization becomes much less trivial. First, new solutions appear when $v>1$ and have negative eigenvalues; thus they are only evanescent. However, as $v$ increases, so do their eigenvalues, which become positive for the retrograde modes (and so the waves become propagative) when $v=(m-k)(m-k-1) / m$ (cf. Lee \& Saio 1997). Second, a large fraction of the modes that exist in the absence of rotation see their eigenvalue grow tremendously with rotation (see Fig. 3). Townsend (2003) has introduced a new classification for modes based on the behavior of their eigenvalues in rapid rotation:

- Gravito-inertial waves: they are similar to normal gravity waves, but modified by the Coriolis acceleration. They are 


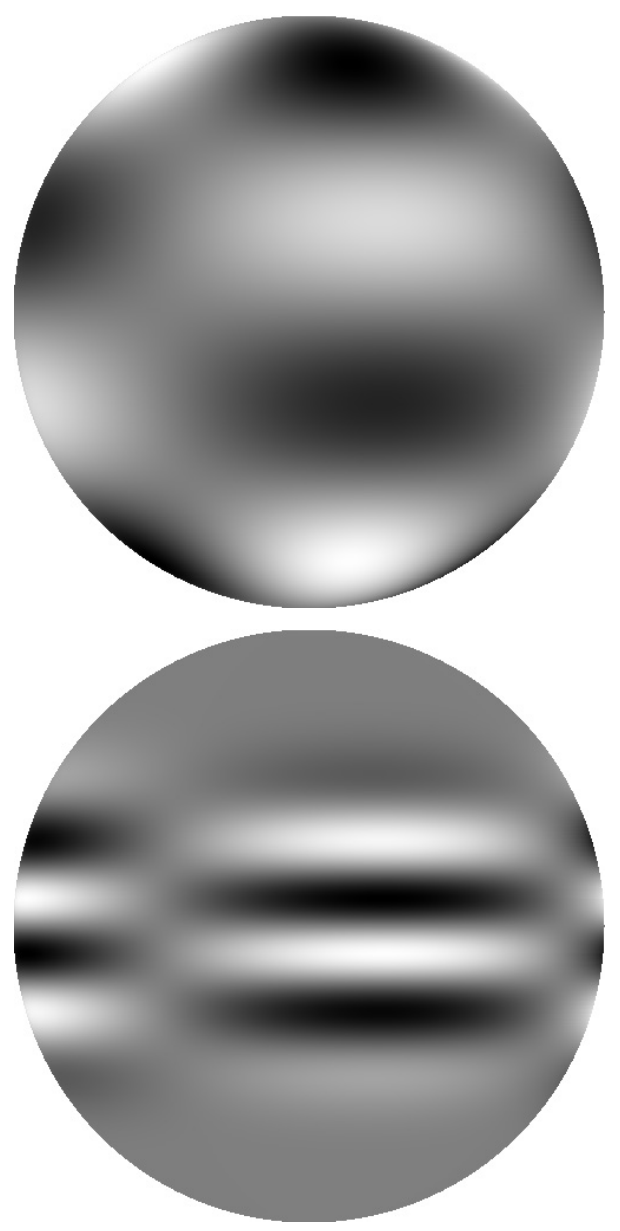

Fig. 2. Top: spherical harmonic $Y_{\ell}^{m}$ with $\ell=5, m=+2$. Bottom: corresponding Hough function $\Theta_{k m} \mathrm{e}^{\mathrm{i} m \phi}$ with $k=+3, m=+2$ and $v \approx 2$.

assigned indexes $s=1,2,3, \ldots$, such that $\Lambda_{s+1, m}(v)>$ $\Lambda_{s m}(v)$.

- Rossby waves: these are purely retrograde waves, which exist only in the case of rapid rotation. They arise from the conservation of specific vorticity, combined with the effect of curvature. They also have indexes $s=1,2,3, \ldots$ but ordered such that $\Lambda_{s+1, m}(v)<\Lambda_{s m}(v)$. The $s=1$ modes are retrograde sectoral waves.

- Yanai waves $^{3}$ : they behave like a mixture of gravity and Rossby waves. The $m \leq 0$ modes exist in the absence of rotation. The $m>0$ modes appear when $v=m+1$ with small eigenvalues, and their horizontal eigenfunctions are then exactly $\Theta(v=m+1)=P_{m+1}^{m}$. When they appear and have small eigenvalues, they behave mostly like Rossby waves; $m \leq 0$ waves and $m>0$ waves with large eigenvalues behave rather like gravity waves. We assign to them index $s=0$.

- Kelvin waves: like Rossby waves, they arise from the conservation of specific vorticity combined this time with the stratification of the medium. They are purely prograde waves, whose characteristics change little with rotation. This occurs because their displacement is very small in the $\theta$ direction. They correspond to the $m<0$ sectoral modes. We assign to them index $s=-1$.

3 They have been named after their discoverer in the Earth's atmosphere, Yanai \& Maruyama (1966).

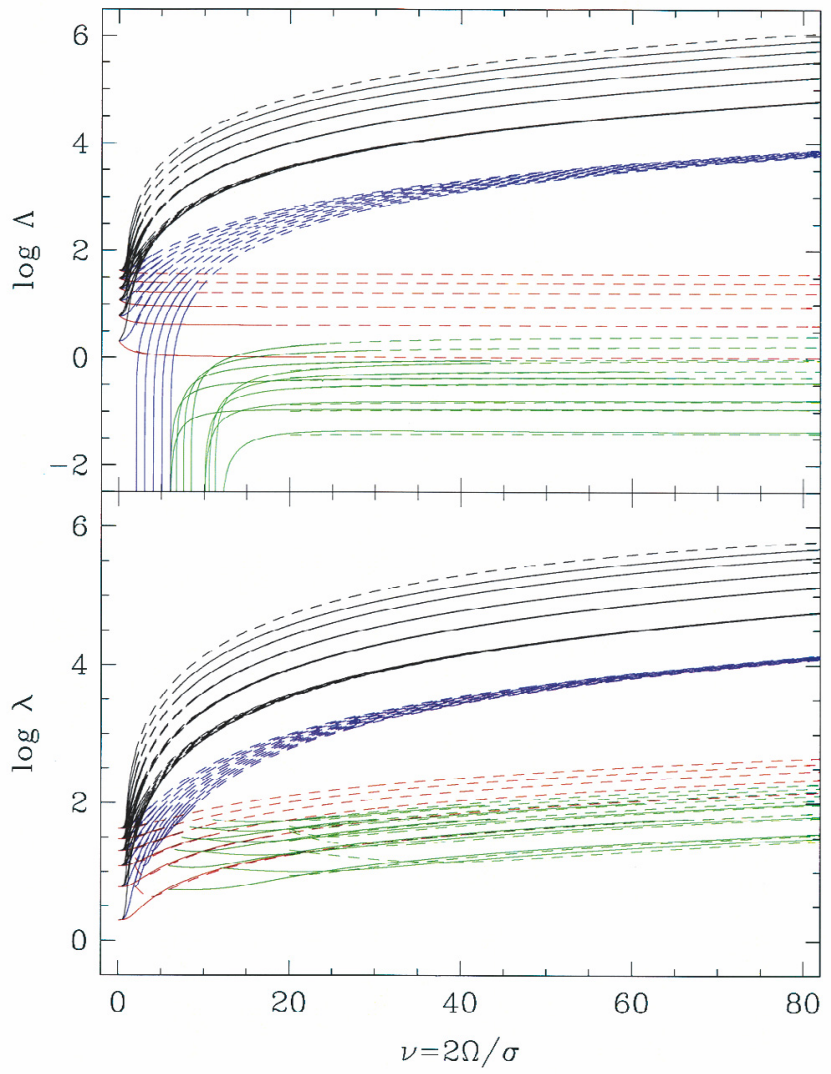

Fig. 3. Eigenvalues $\Lambda$ of Laplace's tidal equation for rapid rotation (top) and equivalent eigenvalues $\lambda$ for the horizontal Laplacian of Hough functions (bottom). Mode classification: black: gravito-inertial waves, blue: Yanai waves, brown: Kelvin waves, green: Rossby waves. Continuous lines correspond to numerical solutions and dashed lines to asymptotic solutions (see Sect. 2.3).

Without rotation, the correspondence between various indexes follows

$s=\ell-m+1$ for $m>0$

and

$s=\ell+m-1$ for $m \leq 0$

and is illustrated in Fig. 4.

\subsection{Horizontal displacement}

Pressure $\left(P^{\prime}\right)$ and density $\left(\rho^{\prime}\right)$ perturbations have a horizontal structure identical to that of the radial displacement (Eq. (7)). This is, however, not the case for the two components of the horizontal displacement, which take the form

$$
\begin{aligned}
& \xi_{\theta}(r, \theta, \phi, t)=\frac{1}{r \sigma^{2}} \frac{P^{\prime}(r)}{\rho} \mathcal{H}^{\theta}(v, \cos \theta) \mathrm{e}^{\mathrm{i} m \phi} \mathrm{e}^{\mathrm{i} \sigma t} \\
& \xi_{\phi}(r, \theta, \phi, t)=\frac{\mathrm{i}}{r \sigma^{2}} \frac{P^{\prime}(r)}{\rho} \mathcal{H}^{\phi}(\nu, \cos \theta) \mathrm{e}^{\mathrm{i} m \phi} \mathrm{e}^{\mathrm{i} \sigma t}
\end{aligned}
$$

with

$$
\begin{aligned}
\mathcal{H}_{s m}^{\theta}(v, \cos \theta)= & \frac{1}{\left(1-v^{2} x^{2}\right) \sqrt{1-x^{2}}} \\
& \times\left[-\left(1-x^{2}\right) \frac{\mathrm{d}}{\mathrm{d} x}+m v x\right] \Theta_{s m}(v, \cos \theta)
\end{aligned}
$$



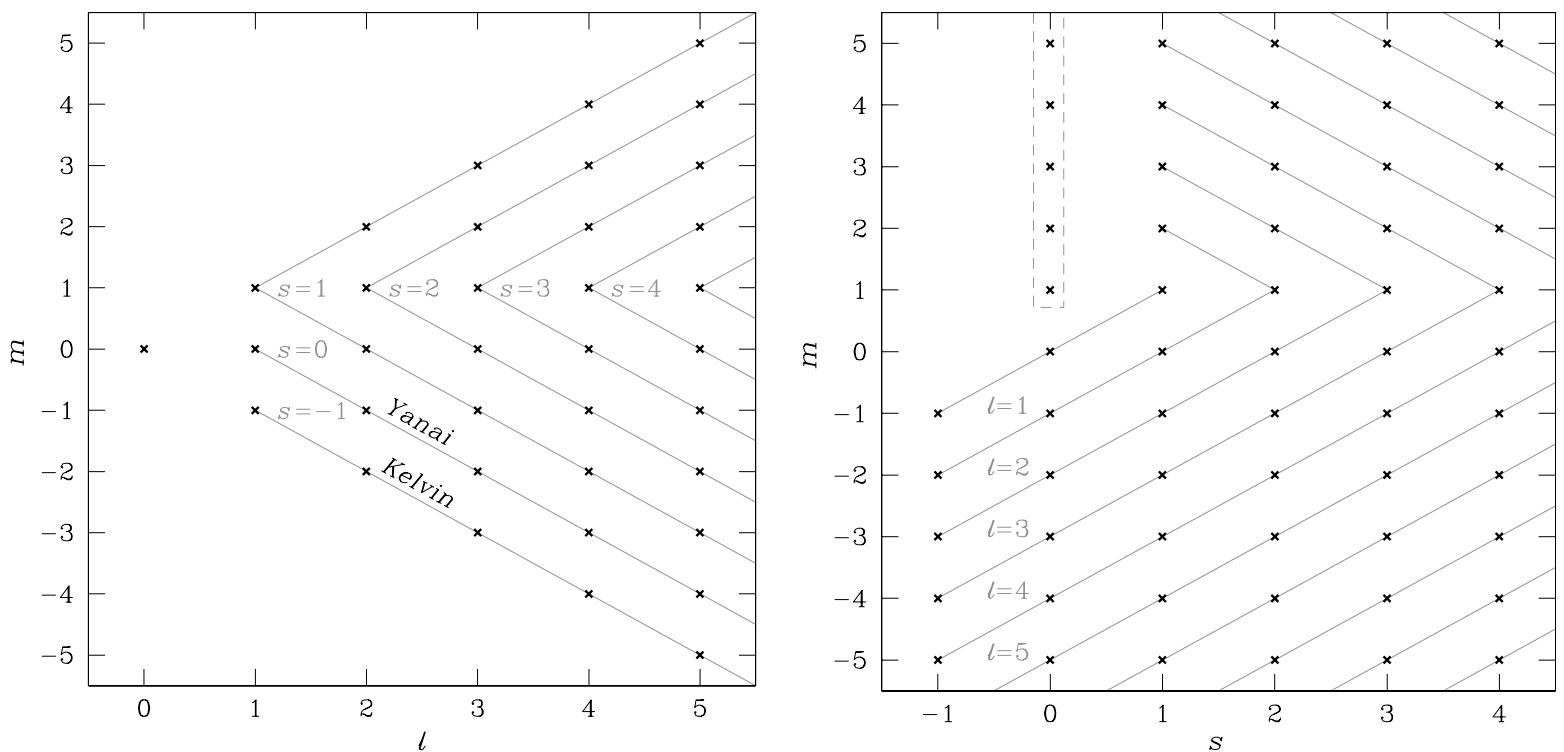

Fig. 4. Correspondence of index $s$ with index $\ell$ in the absence of rotation. Modes in the dashed box (retrograde Yanai waves) do not exist in the absence of rotation.
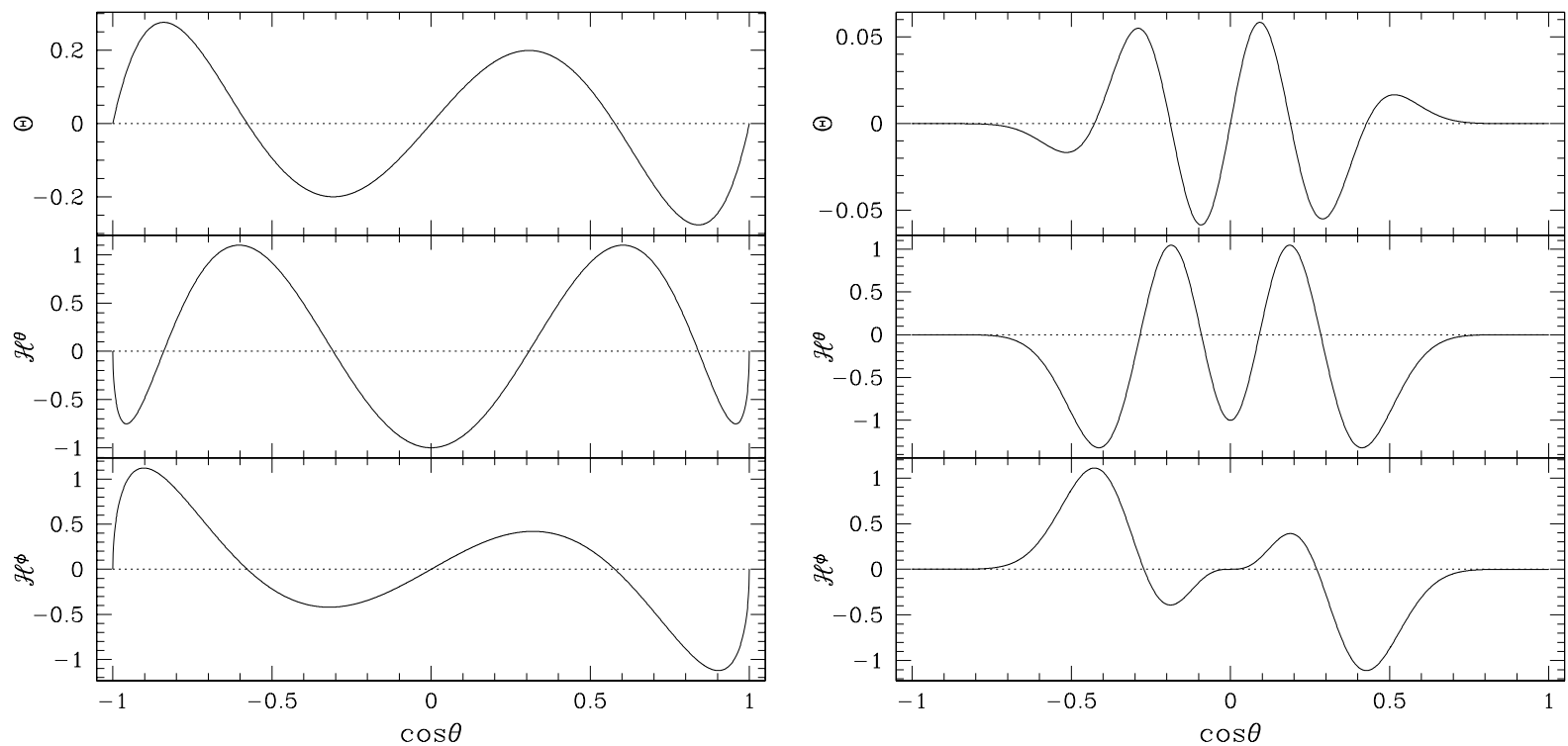

Fig. 5. Hough functions $\Theta_{s m}, \mathcal{H}_{s m}^{\theta}$, and $\mathcal{H}_{s m}^{\phi}$. Normalization is given by $\mathrm{d} \Theta / \mathrm{d} \cos \theta=1$ at $\cos \theta=0$. Left: $s=4, m=2$, and $v=0(\ell=5)$. In this case, $\Theta_{s m}, \mathcal{H}_{s m}^{\theta}$, and $\mathcal{H}_{s m}^{\phi}$ are given by $P_{\ell}^{m}, \mathrm{~d} P_{\ell}^{m} / \mathrm{d} \theta$, and $m P_{\ell}^{m} / \sin \theta$ respectively. Right: $s=4, m=2$, and $v \approx 2$.

$$
\begin{aligned}
\mathcal{H}_{s m}^{\phi}(v, \cos \theta)=\frac{1}{(1-} & \left.v^{2} x^{2}\right) \sqrt{1-x^{2}} \\
& \times\left[-v x\left(1-x^{2}\right) \frac{\mathrm{d}}{\mathrm{d} x}+m\right] \Theta_{s m}(v, \cos \theta)
\end{aligned}
$$

where again $x=\cos \theta$. An example of these functions is shown in Fig. 5. Note that $\Theta_{s m}$ has two more zeros compared to the nonrotating case. Furthermore, $\mathcal{H}_{s m}^{\theta}$ and $\mathcal{H}_{s m}^{\phi}$ are no longer given by simple expressions and have zeros that do not coincide with those of $\Theta_{s m}$.

\subsection{Asymptotic solution}

When considering the impact of IGWs on the distribution of angular momentum within a star, low-frequency waves (with $\sigma \approx 1 \mu \mathrm{Hz}$ ) play a dominant role (this is discussed at length in Talon \& Charbonnel 2005). In the case of massive stars that are generally rapid rotators (with $\Omega \approx 20 \mu \mathrm{Hz}$ ), we are within the limit $v \gg 1$, where asymptotic solutions to Laplace's equation exist (Townsend 2003).

To get these solutions, Townsend defines

$\widehat{\Theta}(\cos \theta)=\sin \theta \mathcal{H}^{\theta}(\cos \theta)$

$\widetilde{\Theta}(\cos \theta)=-\sin \theta \mathcal{H}^{\phi}(\cos \theta)$.

Then, eliminating $\widetilde{\Theta}$, Laplace's equation is equivalent to two coupled first-order equations

$\left[\left(1-x^{2}\right) \frac{\mathrm{d}}{\mathrm{d} x}-m v x\right] \Theta=\left(v^{2} x^{2}-1\right) \widehat{\Theta}$

$\left[\left(1-x^{2}\right) \frac{\mathrm{d}}{\mathrm{d} x}+m v x\right] \widehat{\Theta}=\left[\Lambda\left(1-x^{2}\right)-m^{2}\right] \Theta$. 
Further simplifications to these equations can be made. For gravito-inertial waves and Yanai waves, $\Lambda \gg m^{2}$, while Rossby waves have $\Lambda \ll m^{2}$ (see Fig. 3). When $v \gg 1$, these waves are very confined to the equator, which allows the assumption $\left(1-x^{2}\right) \approx 1$. For Kelvin waves, one has $\Lambda \approx m^{2}$, leading to $|\Theta| \gg|\widehat{\Theta}|$. Townsend (2003) then finds approximate solutions to the Hough functions in the form of Hermite polynomials. We refer the reader to his paper for their derivation. Let us note here that, except for Rossby waves that are less confined to the equator, a direct comparison with the numerical solution for the complete version of Laplace's equation shows that the approximation is valid for $v \gtrsim 2$. For Rossby waves, this approximation becomes valid at much higher values on the order of $v \gtrsim 20$ (see Fig. 3).

In this asymptotic limit, eigenvalues take the form

$$
\Lambda_{s m}(v)=\left[\frac{1}{2} v(2 s+1)+\frac{1}{2} \sqrt{v^{2}(2 s+1)^{2}-4\left(m v-m^{2}\right)}\right]^{2}
$$

for gravito-inertial waves $(s=1,2, \ldots)$,

$$
\Lambda_{s m}(v)=(v-m)^{2}
$$

for Yanai waves $(s=0)$,

$\Lambda_{s m}(v)=m^{2} \frac{2 m v}{2 m v+1}$

for Kelvin waves $(s=-1)$, and

$\Lambda_{s m}(v)=\left[\frac{1}{2} v(2 s+1)-\frac{1}{2} \sqrt{v^{2}(2 s+1)^{2}-4\left(m v-m^{2}\right)}\right]^{2}$

for Rossby waves $(s=1,2, \ldots)$. These asymptotic values are shown in Fig. 3 and are given for $v>2$ for gravito-inertial waves, Yanai waves, and Kelvin waves and for $v>20$ for Rossby waves. As $v$ increases, so does the agreement between the exact and asymptotic value of $\Lambda$.

For gravito-inertial, Yanai and Rossby waves, eigenfunctions are given by

$$
\begin{aligned}
& \Theta(\zeta)=\frac{(\sqrt{\Lambda} v)^{1 / 2}}{\Lambda-m^{2}} \\
& \times\left[s\left(\frac{m}{\sqrt{\Lambda}}+1\right) H_{s-1}(\zeta)+\frac{1}{2}\left(\frac{m}{\sqrt{\Lambda}}-1\right) H_{s+1}(\zeta)\right] \mathrm{e}^{-\zeta^{2} / 2} \\
& \widehat{\Theta}=H_{s}(\zeta) \mathrm{e}^{-\zeta^{2} / 2} \\
& \widetilde{\Theta}(\zeta)=m \frac{(\sqrt{\Lambda} \nu)^{1 / 2}}{\Lambda-m^{2}} \\
& \quad \times\left[s\left(\frac{\sqrt{\Lambda}}{m}+1\right) H_{s-1}(\zeta)+\frac{1}{2}\left(\frac{\sqrt{\Lambda}}{m}-1\right) H_{s+1}(\zeta)\right] \mathrm{e}^{-\zeta^{2} / 2}
\end{aligned}
$$

with $\zeta \equiv \cos \theta(\sqrt{\Lambda} v)^{1 / 2}$ and where $H_{s}$ is the Hermite polynomial of order $s$. For the Kelvin waves, we get

$\Theta(\eta)=\mathrm{e}^{-\eta^{2} / 2}$

$\widehat{\Theta}(\eta)=-\frac{1}{(-m v)^{1 / 2}} \frac{m^{2}}{2 m v+1} \eta \mathrm{e}^{-\eta^{2} / 2}$

$\widetilde{\Theta}(\eta)=-m\left(\frac{\eta^{2}}{2 m v+1}+1\right) \eta \mathrm{e}^{-\eta^{2} / 2}$

with $\eta \equiv \cos \theta(-m v)^{1 / 2}$ (Townsend 2003).

\subsection{Radial dependency}

The formalism used to treat the wave radial dependency is not modified compared to the case of the non-rotating star. In the anelastic approximation $\sigma^{2} \ll N^{2}$, the adiabatic radial displacement $\xi_{r}(r)$ obeys

$\frac{\mathrm{d}^{2} \psi}{\mathrm{d} r^{2}}+\left(\frac{N^{2}}{\sigma^{2}}-1\right) \frac{\Lambda}{r^{2}} \psi=0$

with $\psi(r)=\sqrt{\rho} r^{2} \xi_{r}(r), N^{2}=N_{T}^{2}+N_{\mu}^{2}=\frac{g \delta}{H_{p}}\left(\nabla_{a d}-\nabla\right)+\frac{g \varphi}{H_{p}} \nabla_{\mu}$ and where we neglected the righthand side term (cf. Press 1981). The radial wavenumber is still

$k_{\mathrm{r}}^{2}=\left(\frac{N^{2}}{\sigma^{2}}-1\right) \frac{\Lambda}{r^{2}}$.

However, in contrast to the non-rotating case, the horizontal wave number ${ }^{4}$ is no longer given by $k_{\mathrm{h}}^{2}=\Lambda / r^{2}$. When horizontal gradients are required, an equivalent value has to be taken that we define here as

$\lambda_{s m}^{2}(v)=\frac{\left\langle\left|r^{2} \nabla_{h}^{2} \Theta_{s m}(v)\right|^{2}\right\rangle_{\theta}}{\left\langle\left|\Theta_{s m}(v)\right|^{2}\right\rangle_{\theta}}$

(see Fig. 3 bottom), where $\langle\ldots\rangle_{\theta}$ denotes latitudinal averages. In the absence of rotation, we recover $\lambda_{s m}=\Lambda_{s m}$. We may define an "equivalent" horizontal wave number that is now given by $k_{\mathrm{h}}^{2}=\lambda / r^{2}$, and we have $k_{\mathrm{r}}^{2} \gg k_{\mathrm{h}}^{2}$ as in the non-rotating case (except when $\Lambda \rightarrow 0$ ).

We now apply the WKB method to solve Eq. (31). For a wave propagating towards the surface, we write $\psi(r)=$ $C(r) \mathrm{e}^{-\mathrm{i} \int \mathrm{d} r^{\prime} k_{\mathrm{r}}}$ and get

$\xi_{r}(r) \propto\left|k_{\mathrm{r}}\right|^{-1 / 2} \rho^{-1 / 2} r^{-2} \mathrm{e}^{-\mathrm{i} \int \mathrm{d} r^{\prime} k_{\mathrm{r}}}$.

Assuming that wave damping is dominated by heat diffusion and that this term remains small, the radial displacement becomes $\xi_{r}(r) \mathrm{e}^{-\tau(r) / 2}$ with

$\tau(r)=\int_{r_{\mathrm{c}}}^{r} \mathrm{~d} r^{\prime} \frac{\Lambda^{3 / 2}}{r^{\prime 3}} \frac{K N N_{T}^{2}}{\sigma^{4}} \sqrt{\frac{N^{2}}{N^{2}-\sigma^{2}}}$

(Zahn et al. 1997).

\section{Angular momentum transport}

\subsection{Excitation}

The treatment of wave excitation is more complex in the case of the rotating star. Indeed, the traditional approximation, which requires $\sigma^{2},(2 \Omega)^{2} \ll N^{2}$, is no longer valid in the convection zone; thus, formalisms that convolve the wave eigenfunctions with entropy perturbations and Reynolds stresses in the convection zone (such as those by Goldreich et al. 1994; or Balmforth 1992) require major transformations (Belkacem et al. in prep.) For that reason, we resort to adapting the García López \& Spruit (1991) formalism to our problem.

In that model, waves are excited at the boundary of the radiative zone by the interface deformation caused by convective

${ }^{4}$ In the absence of rotation, Legendre polynomials follow

$$
r^{2} \nabla_{\mathrm{h}}^{2} \Theta=-\Lambda \Theta,
$$

but this is no longer the case when rotation is added (see Eq. (9)). 
eddies. The eddies mean energy density is transfered to waves of the same frequency and with $k_{\mathrm{h}} \leq k_{\text {eddy }}$ according to

$\frac{1}{2} \rho\left\langle v^{2}(\sigma)\right\rangle_{\mathrm{h}}=\left(\frac{k_{\mathrm{h}}}{k_{\text {eddy }}}\right)^{2} \frac{1}{2} \rho\left\langle v_{\text {eddy }}^{2}(\sigma)\right\rangle_{\mathrm{h}}$

(García López \& Spruit 1991), where $\langle\ldots\rangle_{\mathrm{h}}$ denotes horizontal averages. The factor $k_{\mathrm{h}} / k_{\text {eddy }}$ reflects the reduced efficiency in the production of small $k_{\mathrm{h}}$ waves caused by the stochastic nature of turbulent eddies. Assuming a turbulent Kolmogorov spectrum, we have $L_{\text {eddy }} \propto \mathrm{v}_{\text {eddy }}^{3}$ and $L_{\max } \leq L_{\mathrm{c}}$ with $L_{\mathrm{c}}$ the mixing length. The spectral distribution becomes

$k_{\text {eddy }}^{2}(\sigma)=k_{\mathrm{c}}^{2}\left(\frac{\sigma}{\sigma_{\mathrm{c}}}\right)^{3}$

$v_{\text {eddy }}^{2}(\sigma)=v_{\mathrm{c}}^{2}\left(\frac{\sigma}{\sigma_{\mathrm{c}}}\right)^{-1}$

with $\sigma \geq \sigma_{\mathrm{c}}, k_{\mathrm{c}}=2 \pi / L_{\mathrm{c}}$ and where $v_{\mathrm{c}}$ and $\sigma_{\mathrm{c}}$ are the characteristic convective velocities and frequencies, respectively. We get

$\frac{1}{2} \rho\left\langle v^{2}(\sigma)\right\rangle_{\mathrm{h}}=\frac{1}{2} \rho v_{\mathrm{c}}^{2}\left(\frac{k_{\mathrm{h}}}{k_{\mathrm{c}}}\right)^{2}\left(\frac{\sigma}{\sigma_{\mathrm{c}}}\right)^{-4}$.

To proceed further, we assume that the horizontal dependence of IGWs at the radiative boundary is given by Hough functions ${ }^{5}$. Using the "equivalent" eigenvalue given by Eq. (33), one then has

$\frac{1}{2} \rho\left\langle v_{s m}^{2}(\sigma)\right\rangle_{\mathrm{h}}=\frac{\lambda_{s m}}{r_{\mathrm{c}}^{2} k_{\mathrm{c}}^{2}} \frac{1}{2} \rho v_{\mathrm{c}}^{2}\left(\frac{\sigma}{\sigma_{\mathrm{c}}}\right)^{-4}$.

An alternative definition of the equivalent eigenvalue $\lambda=$ $\left\langle\left|r^{2} \nabla_{\mathrm{h}}^{2} \Theta\right|\right\rangle_{\theta} /\langle|\Theta|\rangle_{\theta}$ leads to very similar results. Following Mathis (2005), one could also use a projection of the Hough functions onto spherical harmonics for which horizontal wavenumbers are well-defined. However, in the case of fast rotation numerical problems are encountered with this last method because Legendre polynomials are not a good basis for projecting Hermite polynomials.

\subsection{Energy flux}

Angular momentum transport by IGWs is dominated by the lowfrequency waves. Here, we will thus adopt $\sigma^{2} \ll N^{2}$ and use $k_{\mathrm{r}}^{2}=(N / \sigma)^{2} \Lambda / r^{2}$. The kinetic energy flux per unit frequency is given by

$\mathcal{F}_{E}=\frac{1}{2} \rho\left\langle v^{2}\right\rangle_{\mathrm{h}} v_{\mathrm{g}}$,

where the radial group velocity $v_{\mathrm{g}}$ is given by

$v_{\mathrm{g}}=\frac{\mathrm{d} \sigma}{\mathrm{d} k_{\mathrm{r}}}=-\frac{\sigma}{k_{\mathrm{r}}}$

In the case of main sequence massive stars, we have $k_{\mathrm{r}}<0$, corresponding to waves traveling from the convective core to the

5 This is formally wrong since at this boundary we have $N^{2} \longrightarrow 0$, which contradicts the traditional approximation. However, as $N^{2}$ rises rapidly away from the boundary, and considering the inescapable presence of a slight amount of overshooting, the approximation is acceptable.

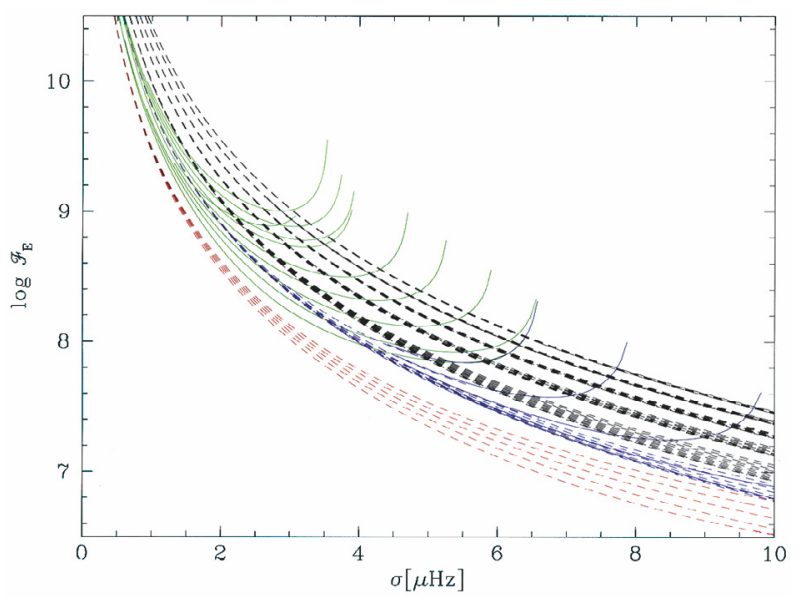

Fig. 6. Energy flux in IGWs at the base of radiative envelope in a $3 M_{\odot}$ main sequence star with $X_{\mathrm{c}}=0.5$. Black: gravito-inertial waves, blue: Yanai waves, brown: Kelvin waves, green: Rossby waves. Continuous lines correspond to numerical solutions and dashed lines, to asymptotic solutions (see Sect. 2.3).

surface. Using Eq. (40) to get the energy of a given mode, we obtain

$\mathcal{F}_{E}(\sigma, s, m)=\frac{\rho \lambda_{s m}}{2 \sqrt{\Lambda_{s m}}} \frac{v_{\mathrm{c}}^{2} \sigma_{\mathrm{c}}^{2}}{r_{\mathrm{c}} k_{\mathrm{c}}^{2} N_{\mathrm{c}}}\left(\frac{\sigma}{\sigma_{\mathrm{c}}}\right)^{-2}$,

where $N_{\mathrm{c}}$, which would formally be 0 at the interface, is taken a fraction of a pressure scale height into the radiative region.

These fluxes have been computed for a $3 M_{\odot}$ main-sequence, population I star calculated with the Geneva stellar evolution code and for a rotation velocity of $\Omega=20 \mu \mathrm{Hz}$ (Fig. 6). This value, typical of massive stars, corresponds to $\sim 40 \%$ of the surface critical velocity.

In the case of Rossby and retrograde Yanai waves, one can see that they appear with a significant energy flux. This is caused by the divergence of the group velocity when $\Lambda_{s m}$ is close to 0 (see Eq. (42)). This situation is unphysical; however, it will be compensated for by a term in $\Lambda$ in the calculation of the angular momentum flux (Eq. (48)). Thus we do not need to correct this in the present treatment.

\subsection{Angular momentum flux}

We must now convert the energy flux into an angular momentum flux. Following Zahn et al. (1997), we write

$\mathcal{F}_{E}=\frac{1}{2} \rho\left\langle v_{\mathrm{r}}^{2}+v_{\theta}^{2}+v_{\phi}^{2}\right\rangle_{\mathrm{h}} v_{\mathrm{g}}$,

with the real part of the velocity field $\boldsymbol{v}=\mathrm{i} \sigma \boldsymbol{\xi}$

$v_{\mathrm{r}}=A(r) \sin \left(\sigma t-\int_{r_{\mathrm{c}}}^{r} \mathrm{~d} r^{\prime} k_{\mathrm{r}}+m \phi\right) \Theta(v, \cos \theta) \mathrm{e}^{-\tau(r) / 2}$

$v_{\theta}=-\frac{r k_{\mathrm{r}}}{\Lambda} A(r) \cos \left(\sigma t-\int_{r_{\mathrm{c}}}^{r} \mathrm{~d} r^{\prime} k_{\mathrm{r}}+m \phi\right) \mathcal{H}^{\theta}(v, \cos \theta) \mathrm{e}^{-\tau(r) / 2}$

$v_{\phi}=\frac{r k_{\mathrm{r}}}{\Lambda} A(r) \sin \left(\sigma t-\int_{r_{\mathrm{c}}}^{r} \mathrm{~d} r^{\prime} k_{\mathrm{r}}+m \phi\right) \mathcal{H}^{\phi}(\nu, \cos \theta) \mathrm{e}^{-\tau(r) / 2}$,

given by the WKB method for $v_{r}$, and with help of the continuity equation in the Boussinesq approximation for $v_{\theta}$ and $v_{\phi}$. The 


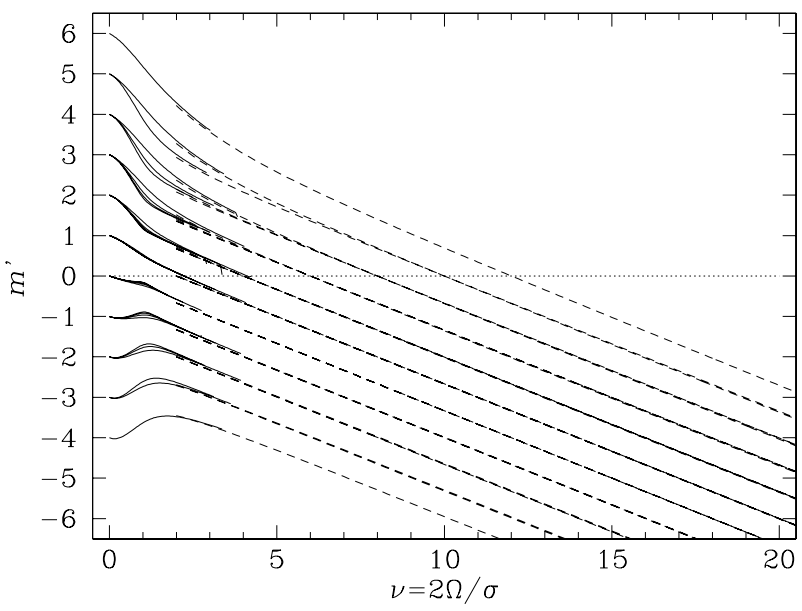

Fig. 7. Ratio $m^{\prime}=-\frac{\sigma}{2} \frac{\mathcal{F}_{J}}{\mathcal{F}_{E}}$ for the gravito-inertial waves when the Coriolis acceleration is neglected in the calculation of the angular momentum flux. Continuous lines correspond to numerical solutions and dashed lines to asymptotic solutions (see Sect. 2.3).

energy flux for a given mode becomes

$\mathcal{F}_{E}=-\frac{A^{2}(r)}{4} \rho\left(\left\langle|\Theta|^{2}\right\rangle_{\theta}+\frac{r^{2} k_{\mathrm{r}}^{2}}{\Lambda^{2}}\left[\left\langle\left|\mathcal{H}^{\theta}\right|^{2}\right\rangle_{\theta}+\left\langle\left|\mathcal{H}^{\phi}\right|^{2}\right\rangle_{\theta}\right]\right) \frac{\sigma}{k_{\mathrm{r}}} \mathrm{e}^{-\tau(r)}$.

This equation is equivalent to the one obtained by Mathis (2005).

In the absence of rotation, the angular momentum flux is expressed as (Zahn et al. 1997)

$\mathcal{F}_{J}=\left\langle\rho r \sin \theta v_{\mathrm{r}} v_{\phi}\right\rangle_{\mathrm{h}}$,

which becomes

$\mathcal{F}_{J}=\frac{A^{2}(r)}{2} \rho r\left\langle\sin \theta \Theta \mathcal{H}^{\phi}\right\rangle_{\theta} \frac{r k_{\mathrm{r}}}{\Lambda} \mathrm{e}^{-\tau(r)}$

for a given mode. When this simple formulation is applied to gravito-inertial waves, one finds that the sign of angular momentum transport varies with $v$ (see Fig. 7). As the rotation parameter $v$ increases, the value $m^{\prime}=-\frac{\sigma}{2} \frac{\mathcal{F}_{J}}{\mathcal{F}_{E}}$ diminishes linearly and changes sign for $v=2 m$. This would imply that a retrograde wave $(m>0)$ would, for fast enough rotation, carry a positive angular momentum flux $\left(m^{\prime}<0\right)$. This can be understood with the aid of Fig. 8. In the absence of rotation, $\Theta=P_{\ell}^{m}$ and $\mathcal{H}^{\phi}=m P_{\ell}^{m} / \sin \theta$; their product always has the sign of $m$. This is no longer the case when the Coriolis acceleration is included. The integral $\left\langle\sin \theta \Theta \mathcal{H}^{\phi}\right\rangle_{\theta}$ may thus change sign.

The solution to this paradox lies in the fact that, in a rotating system, the angular momentum flux is actually given by

$\mathcal{F}_{J}=\left\langle\rho r \sin \theta v_{\mathrm{r}}\left(v_{\phi}+2 \Omega \cos \theta \xi_{\theta}\right)\right\rangle_{\mathrm{h}}$

(Jones 1967; Bretherton 1969). The first term in this equation corresponds to the angular momentum flux across an Eulerian surface, and the second term to a flux associated with a Lagrangian contribution to angular momentum (Bretherton 1969). In that case, the actual angular momentum flux thus becomes

$\mathcal{F}_{J}=\frac{A^{2}(r)}{2} \rho r\left(\left\langle\sin \theta \Theta \mathcal{H}^{\phi}\right\rangle_{\theta}-v\left\langle\sin \theta \cos \theta \Theta \mathcal{H}^{\theta}\right\rangle_{\theta}\right) \frac{r k_{\mathrm{r}}}{\Lambda} \mathrm{e}^{-\tau(r)}$ for a given mode. The ratio between the kinetic energy and the angular momentum flux is thus

$\frac{\mathcal{F}_{J}}{\mathcal{F}_{E}}=-\frac{2 \Lambda}{\sigma} \frac{\left\langle\sin \theta \Theta \mathcal{H}^{\phi}\right\rangle_{\theta}-v\left\langle\sin \theta \cos \theta \Theta \mathcal{H}^{\theta}\right\rangle_{\theta}}{\left\langle\left|\mathcal{H}^{\theta}\right|^{2}\right\rangle_{\theta}+\left\langle\left|\mathcal{H}^{\phi}\right|^{2}\right\rangle_{\theta}}$,

where we use the approximation $r^{2} k_{\mathrm{r}}^{2} \gg \Lambda^{2}$. In the absence of rotation, we get

$\frac{\mathcal{F}_{J}}{\mathcal{F}_{E}}=-\frac{2 m}{\sigma}$.

We define an equivalent azimuthal number $m^{\prime}$ such that

$m^{\prime} \equiv-\frac{\sigma}{2} \frac{\mathcal{F}_{J}}{\mathcal{F}_{E}}=\Lambda \frac{\left\langle\sin \theta \Theta \mathcal{H}^{\phi}\right\rangle_{\theta}-v\left\langle\sin \theta \cos \theta \Theta \mathcal{H}^{\theta}\right\rangle_{\theta}}{\left\langle\left|\mathcal{H}^{\theta}\right|^{2}\right\rangle_{\theta}+\left\langle\left|\mathcal{H}^{\phi}\right|^{2}\right\rangle_{\theta}}$

which has been evaluated numerically and is shown in Fig. 9.

In the case of gravito-inertial waves, as $v$ increases, the value $m^{\prime}$ rapidly converges towards $m / 3$, for all orders $s$. This implies that the symmetry that exists between prograde and retrogrades waves is conserved in the rotating case ${ }^{6}$. Yanai waves behave similarly, but with a slower convergence rate.

In the case of the Kelvin waves, $m^{\prime}$ varies only slightly with rotation and remains close to $m$. Their angular momentum flux is always positive. Rossby waves appear with $m^{\prime} \simeq 0$, and its value rises slowly and tends towards $m$ with the increase in the rotation rate. Their angular momentum flux is always negative.

\subsection{Angular momentum deposition}

The angular momentum distribution within the star evolves under the effect of the damping of IGWs. When traveling inwards, each wave deposits its angular momentum at the location where it is damped. In stars, the major source of damping is thermal diffusivity (cf. Sect. 2.4), which is different for each wave. We define the angular momentum luminosity $\mathcal{L}_{J}=4 \pi r^{2} \mathcal{F}_{J}$, which in the WKB approximation can be expressed as

$\mathcal{L}_{J}(r)=4 \pi r^{2} \mathcal{F}_{J}\left(r_{\mathrm{c}}\right) \mathrm{e}^{-\tau(r)}$.

Then, the local angular momentum evolves according to

$\frac{\mathrm{d}}{\mathrm{d} t}\left(\frac{8 \pi}{3} \rho \Omega r^{4}\right)=-\frac{\mathrm{d}}{\mathrm{d} r}\left(\int \mathrm{d} \sigma \sum_{s, m} \mathcal{L}_{J}(\sigma, s, m, r)\right)$

(Zahn et al. 1997).

In this exploratory step, we examine where damping occurs for each type of wave. Let us first look at Fig. 10, which gives the local angular momentum luminosity and the angular momentum deposition $-\mathrm{d} \mathcal{L}_{J} / \mathrm{d} r$ of waves with a frequency $\sigma=3 \mu \mathrm{Hz}$, for the $3 M_{\odot}$ model of Fig. 6 (the rotation parameter is $v=13.3$ ). Since all waves shown here have the same frequency, the location of the deposition is determined by the eigenvalue $\Lambda$ (see Eq. (35)). The rapid fall in the local amplitude at the convective boundary (over $\delta r \simeq 1 \%$ ) is caused by the mean molecular weight gradient that has been left behind the regressing core, and damping increases in the outer region with the thermal diffusivity $K$.

\footnotetext{
${ }^{6}$ Note however that excitation could be asymmetric.
} 

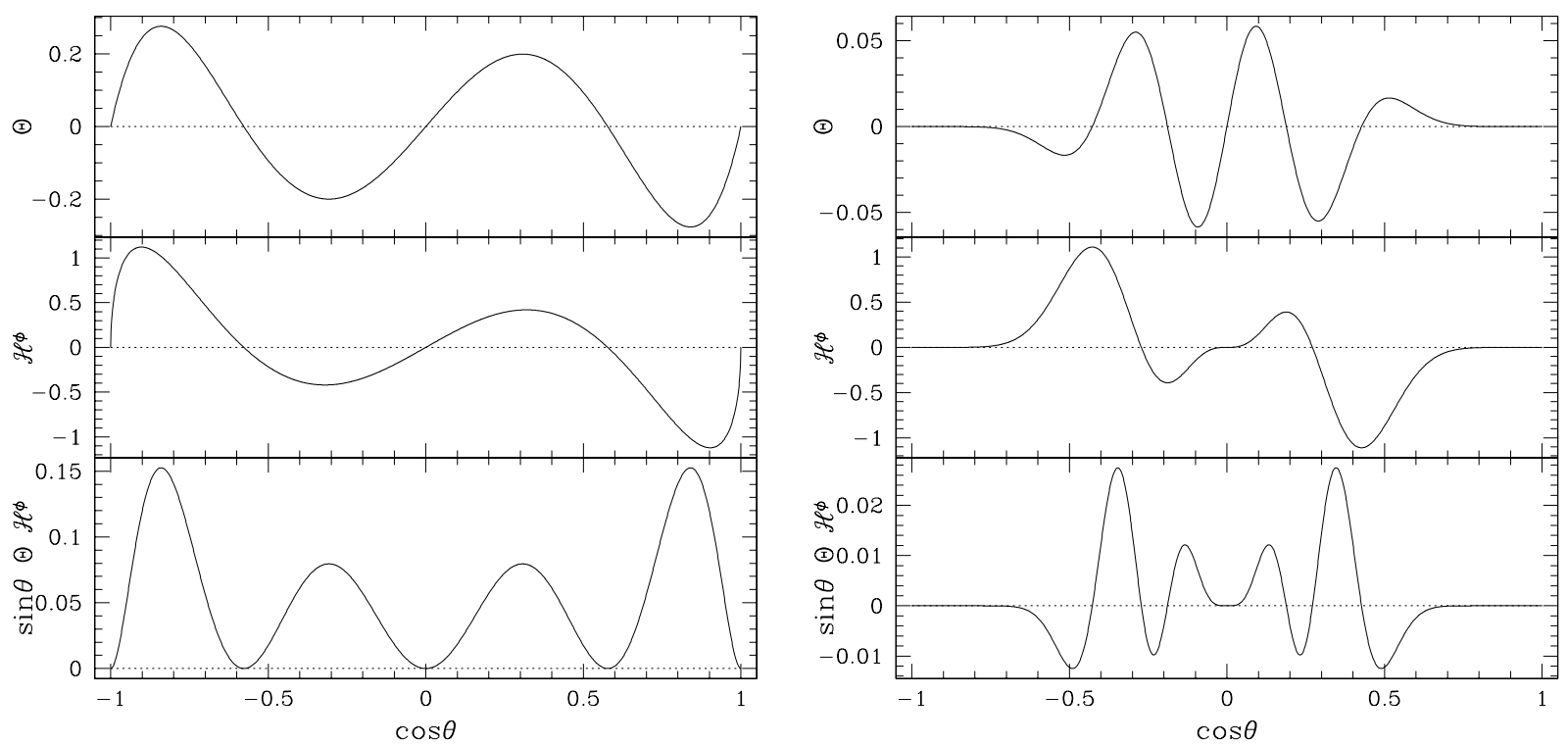

Fig. 8. Left: hough functions $\Theta_{s m}$ and $\mathcal{H}_{s m}^{\phi}$ for $s=4, m=2(\ell=5), v=0$, and their product $\sin \theta \Theta \mathcal{H}^{\phi}$, which is used for the angular momentum flux. Normalization is given by $\mathrm{d} \Theta / \mathrm{d} \cos \theta=1$ at $\cos \theta=0$. Right: equivalent Hough functions for $v \simeq 2$. In the rotating case, the product $\sin \theta \Theta \mathcal{H}^{\phi}$ changes signs locally.
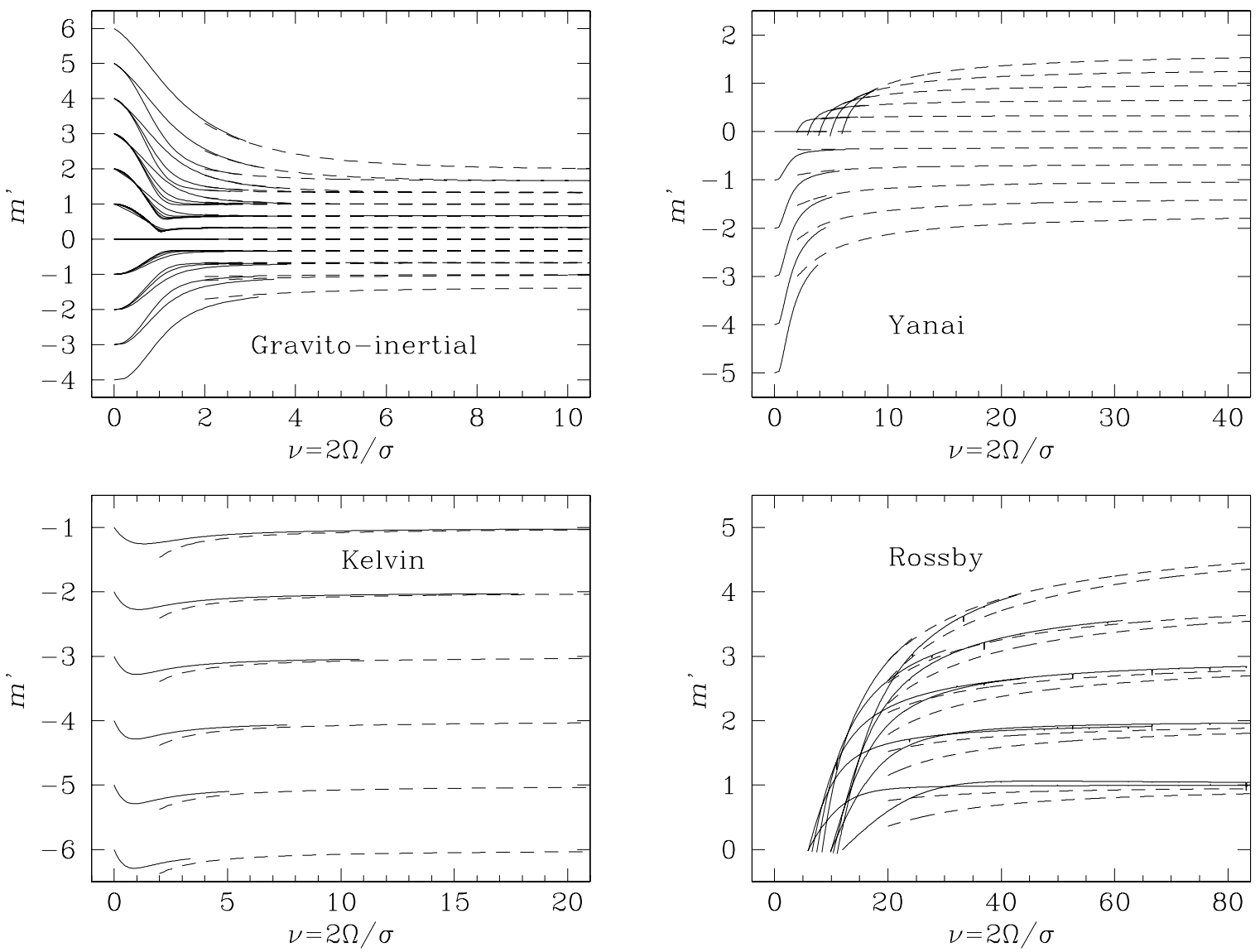

Fig. 9. Ratio $m^{\prime}=-\frac{\sigma}{2} \frac{\mathcal{F}_{J}}{\mathcal{F}_{E}}$ for various modes. Top left: gravito-inertial waves corresponding to $\ell \leq 6$. Top right: Yanai waves of order $m=$ $-5, \ldots,+3$. Bottom left: Kelvin waves of order $m \geq-6$. Bottom right: Rossby waves of order $s=1,2$, and $m \leq 4$. Continuous lines correspond to numerical solutions and dashed lines to asymptotic solutions (see Sect. 2.3).

\section{Discussion}

In this paper, we have examined the transport of angular momentum by low-frequency waves excited by core convection. The traditional approximation was used to evaluate the impact of the Coriolis acceleration on waves. We find two main effects:

- the horizontal structure, and hence the amount of angular momentum carried by a wave, is modified;

- new types of waves appear. 


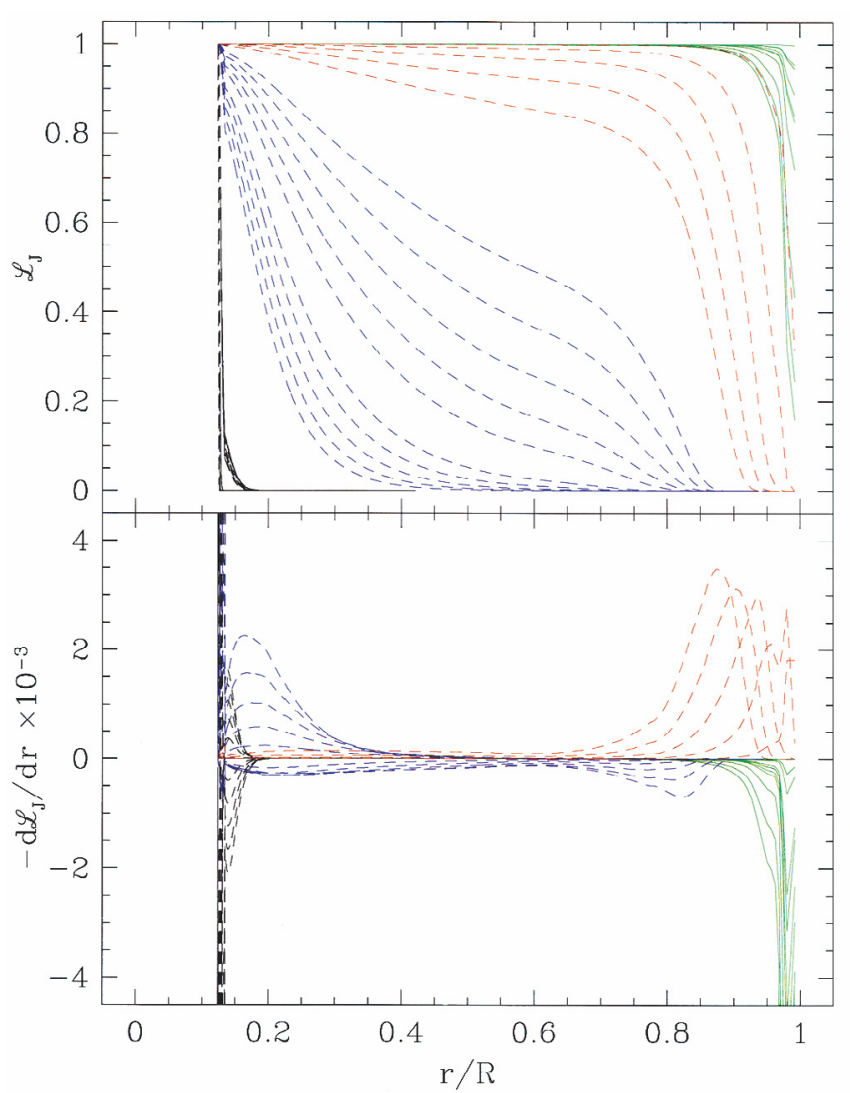

Fig. 10. Local angular momentum luminosity normalized to 1 at the base of the radiative zone (top) and local deposition of angular momentum (bottom) for waves with $\sigma=3 \mu \mathrm{Hz}$ in a $3 M_{\odot}$ main sequence $\operatorname{star}\left(X_{\mathrm{c}}=0.5\right)$. Black: gravito-inertial waves; blue: Yanai waves; brown: Kelvin waves; green: Rossby waves. Continuous lines correspond to numerical solutions and dashed lines to asymptotic solutions (see Sect. 2.3).

In the case of gravito-inertial waves, the main effect of the Coriolis acceleration is to confine the wave towards the equator. This reduces the total angular momentum carried by a wave of a given amplitude and frequency (Fig. 9). As rotation increases, a saturation exists in this reduction, which tends towards $m^{\prime}=-\frac{\sigma}{2} \frac{\mathcal{F}_{J}}{\mathcal{F}_{E}}=\frac{m}{3}$. Rotation also increases their radial wave number (see Eq. (32) and Fig. 3), and hence their damping. These waves are thus deposited very close to the convection core (see Fig. 10). If the asymmetry in the excitation of prograde and retrograde waves is not too strong, we expect that the damping of these waves could produce a shear layer oscillation (SLO) similar to the one present in slowly rotating stars (Talon \& Charbonnel 2005, and references therein).

The Yanai waves, whose main restoring force is also gravity for high values of the rotation parameter $v=2 \Omega / \sigma$, have the same limit value for $m^{\prime}$. Their eigenvalues $\Lambda$ are, however, lower so they are damped farther from the convection core (Fig. 10) and over a larger portion of the star. These most certainly do not produce a second SLO but could generate a local shear in the interior (corresponding to the stationary solution of Kim \& MacGregor 2001).

The other two types of waves, namely Kelvin and Rossby waves, show a somewhat different behavior. For these waves, the main restoring force is the conservation of vorticity, combined with stratification in the first case and in the second, combined with curvature. These two types of waves have the same limiting value $m^{\prime}=m$, and their eigenvalues $\Lambda$ are lower than those of both gravito-inertial and Yanai waves. As a result, they are damped much closer to the stellar surface (Fig. 10). These waves could create a strong shear in that region and could thus induce a large amount of mixing close to the stellar surface. Complete dynamical simulations remain to be completed to verify these conjectures.

\section{References}

Balmforth, N. J. 1992, MNRAS, 255, 639

Berthomieu, G., Gonczi, G., Graff, P., Provost, J., \& Rocca, A. 1978, A\&A, 70, 597

Bildsten, L., Ushomirsky, G., \& Cutler, C. 1996, ApJ, 460, 827

Bretherton, F. P. 1969, Quart. J. R. Met. Soc., 95, 213

Charbonnel, C., \& Talon, S. 2005, Science, 309, 2189

Cowling, T. G. 1941, MNRAS, 101, 367

Daszyńska-Daszkiewicz, J., Dziembowski, W. A., \& Pamyatnykh, A. A. 2007, Acta Astron., submitted

Eckart, C. 1960, Hydrodynamics of Oceans and Atmospheres (Oxford: Pergamon Press)

García López, R. J., \& Spruit, H. C. 1991, ApJ, 377, 268

Goldreich, P., Murray, N., \& Kumar, P. 1994, ApJ, 424, 466

Jones, W. L. 1967, J. Fluid Mech., 30, 439

Kim Eun-jin, \& MacGregor, K. B. 2001, ApJ, 556, L117

Kumar, P., \& Quataert, E. J. 1997, ApJ, 475, L143

Kumar, P., Talon, S., \& Zahn, J.-P. 1999, ApJ, 520, 859

Heger, A., Woosley, S. E., \& Langer, N. 2000, NewAR, 44, 297

Hough, S. S. 1898, Phil. Trans. R. Soc. 191A, 139

Lee, U., \& Saio, H. 1997, ApJ, 491, 839

Longuet-Higgins, F. R. S. 1968, Phil. Trans. R. Soc. 262A, 511

Maeder, A., \& Meynet, G. 2000, ARA\&A, 38, 143

Mathis, S. 2005, Ph.D. Thesis, Université Paris VII

Press, W. H. 1981, ApJ, 245, 286

Press, W. H., Teukolsky, S. A., Vetterling, W. T., \& Flanneray, B. P. 1992, Numerical Recipes in FORTRAN, The art of scientific computing, 2nd edn. (Cambridge University Press)

Schatzman, E. 1993, A\&A, 279, 431

Talon, S. 1997, Ph.D. Thesis, Université Paris VII

Talon, S., \& Charbonnel, C. 1998, A\&A, 335, 959

Talon, S., \& Charbonnel, C. 2003, A\&A, 405, 1025

Talon, S., \& Charbonnel, C. 2005, A\&A, 440, 981

Talon, S., Zahn, J.-P., Maeder, A., \& Meynet, G. 1997, A\&A, 322, 209

Townsend, R. H. D. 2003, MNRAS, 340, 1020

Unno, W., Osaki, Y., Ando, H., Saio, H., \& Shibahashi, H. 1989, Nonradial oscillations of stars, 2nd edn. (University of Tokyo Press)

Wallerstein, G., Herbig, G. H., \& Conti, P. S. 1965, ApJ, 141, 610

Yanai, M., \& Maruyama, T. 1966, J. Meteorol. Soc. Japan, 44, 291

Zahn, J.-P., Talon, S., \& Matias, J. 1997, A\&A, 322, 320 